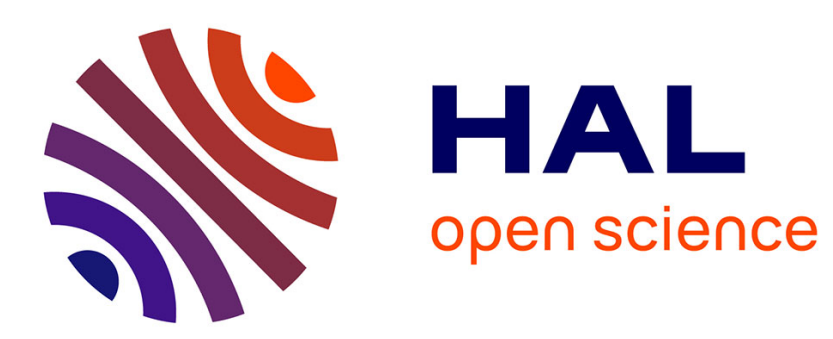

\title{
Stereo-image Rectification for Dense 3D Reconstruction in Scanning Electron Microscope
}

Andrey Kudryavtsev, Sounkalo Dembele, Nadine Piat

\section{To cite this version:}

Andrey Kudryavtsev, Sounkalo Dembele, Nadine Piat. Stereo-image Rectification for Dense 3D Reconstruction in Scanning Electron Microscope. International Conference on Manipulation, Automation and Robotics at Small Scales, Jul 2017, Montréal, Canada. hal-02868209

\section{HAL Id: hal-02868209 \\ https://hal.science/hal-02868209}

Submitted on 15 Jun 2020

HAL is a multi-disciplinary open access archive for the deposit and dissemination of scientific research documents, whether they are published or not. The documents may come from teaching and research institutions in France or abroad, or from public or private research centers.
L'archive ouverte pluridisciplinaire $\mathbf{H A L}$, est destinée au dépôt et à la diffusion de documents scientifiques de niveau recherche, publiés ou non, émanant des établissements d'enseignement et de recherche français ou étrangers, des laboratoires publics ou privés. 


\title{
Stereo-image Rectification for Dense 3D Reconstruction in Scanning Electron Microscope
}

\author{
Andrey V. Kudryavtsev, Sounkalo Dembélé and Nadine Piat \\ FEMTO-ST, AS2M, Univ. Bourgogne Franche-Comté, \\ Univ. de Franche-Comté/CNRS/ENSMM, \\ 24 rue Savary, F-25000 Besançon, France \\ Email: firstname.familyname@femto-st.fr
}

\begin{abstract}
Stereo rectification is a crucial step for a number of computer vision problems and in particular for dense 3D reconstruction which is a very powerful characterization tool for microscopic objects. Rectification simplifies and speeds up the correspondence search in a pair of images: the search space is reduced to a horizontal line. It is mainly developed for perspective camera model: a projective transformation is found and applied to both images. This paper addresses the rectification problem for an image pair obtained with Scanning Electron Microscope (SEM). In this case, image formation is described by a parallel projection, indeed perspective effects can be neglected because of the low value of the sample size over working distance ratio. Based on these hypotheses, a robust estimation of the fundamental matrix, that describes the geometry of the image pair, is proposed. It filters out up to $\mathbf{5 0} \%$ of outliers in a feature correspondence set. Then, the matrix is used to develop a rectification solution: the problem is reduced to two rotations about the optical axis of the camera. The solution is accurate and does not require any calibration of SEM. It is validated with two image pairs from two different field effect SEMs.
\end{abstract}

\section{INTRODUCTION}

Dense 3D reconstruction is a very powerful tool allowing the characterization of the microscopic objects, allowing to measure 3D object properties such as angles, surfaces or volumes that cannot be directly retrieved from images. Moreover, 3D reconstruction provides the three dimensional range information that helps users to perform micromanipulation tasks. The crucial step towards dense reconstruction is the search for correspondence for every pixel in a stereo image. Algorithms such as SIFT [1] and SURF [2] with further matching allow to obtain only a sparse correspondence set which is not enough in many cases. Another option is the realization of dense matching, which consists in a search of correspondence for every pixel of the first image in the second one to build a disparity map to recover the depth variation. In [3], the 3D reconstruction in SEM is estimated from two images obtained by tilting the object exactly about the vertical axis of the image. However, when it is not true, the task of dense matching becomes numerically complicated because the search space of correspondence represents the entire image. Nonetheless, it can be simplified by using various techniques of rectification.

Rectification consists in warping two images in the common plane (making them coplanar) to reduce the search of correspondence to one dimension, i.e., to a horizontal line.
This technique is based on epipolar geometry which gives a number of geometric constraints between the 3D points and their projections onto the 2D images. These constraints can be rewritten mathematically in the form of $3 \times 3$ fundamental matrix. Usually, these constraints are based on the assumption that camera model is perspective [4], [5] and then the goal of rectification consists in applying a pair of projective transformations to the image pair. However, the model and then rectification can be simplified regarding special imaging conditions, e.g., when the object is far away from the view point, i.e., when the focal length is much bigger than the depth variation of the object which is the case in Scanning Electron Microscope (SEM). For SEM, the perspective effects can be neglected and a parallel projection model can be used for magnification values bigger than $\times 1000$, which is confirmed in the literature [6], [7], [8]. Such model assumes that all projection rays are parallel, which means that all epipolar lines are parallel and the epipoles are at infinity [9].

Basically, rectification algorithms can be subdivided into two main classes depending on whether the cameras are calibrated [10] or not [11]. In the case of parallel projection, the calibration is the subject of finding eight parameters, corresponding to eight degrees of freedom. Even if this subject is well studied, the SEM intrinsic calibration can be very complex due to the following reasons. Firstly, in most cases it requires a calibration template, which often means a special step of fabrication of such object, which can be very expensive and time consuming. Moreover, it is very difficult to guarantee the quality of its fabrication, which has a profound impact on the precision of the feature extraction algorithms. Secondly, the classic calibration [12] needs to be done offline, which can be very restrictive in some applications where the calibration object cannot be placed in front of the camera once the operation started. Thirdly, which includes partially the second point, there is a problem of maintainability of calibration parameters. In order to re-calibrate the camera, the main operation task should be stopped. Thus, in this paper we opt for the uncalibrated rectification.

The problem of rectification for parallel projection was partially adressed in [13], [4]. Authors work with perspective cameras and separate the task of rectification on two transformations: projective and affine. They firstly find a projective transformation in order to reduce the rectification task to an 

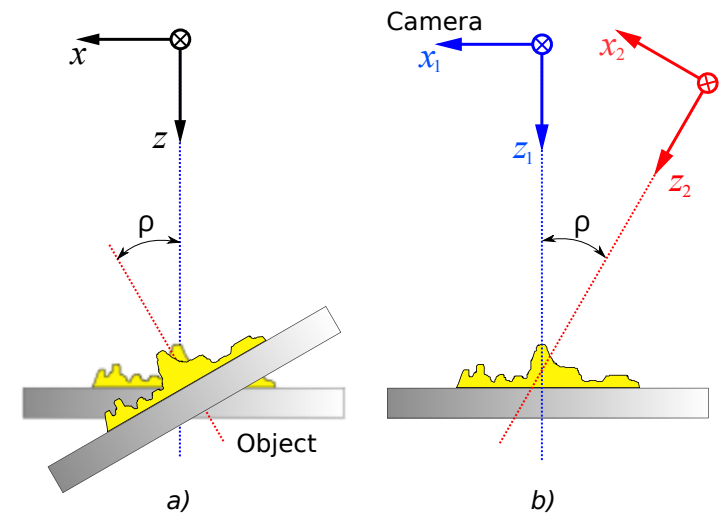

Fig. 1. Equivalence between object motion and camera motion. a) object is moving, camera is static, b) camera is moving, object is static.

affine one. The affine transformation represents scale, rotation and translation. All of these parameters are then found by using optimization approaches.

Our contribution consists in the development of a direct linear rectification method for uncalibrated SEM. At our knowledge, this topic has never been addressed in the literature. The method is based on epipolar geometry, on the special form of the fundamental matrix in case of parallel projection. A robust algorithm based on Least Median of Squares is used for estimation of the affine fundamental matrix for a pair of uncalibrated images. The rectifying transformation is then directly derived from the elements of this matrix. The image pair is obtained by moving the robot stage inside SEM. However, we consider that the object was static and that the camera (SEM) performed the motion. It can be seen from Fig. 1 that these situations are equivalent.

The remaining of this paper is organized as follows. Section II presents the camera model and its properties. In Section III, a method for robust estimation of fundamental matrix is presented as well as the rectification algorithm. Section IV shows the results of algorithm application.

\section{PARAllel PROJECTION MODEL}

The parallel projection of a $3 \mathrm{D}$ point $\mathbf{Q}=\left(Q_{x}, Q_{y}, Q_{z}, 1\right)^{\top}$ (in homogeneous coordinates) to the image frame can be written as follows:

$$
\mathbf{q}=\mathbf{K} \boldsymbol{\Pi}^{c} \mathbf{T}_{w} \mathbf{Q}
$$

with $\mathbf{K}=\left(\begin{array}{ccc}f_{x} & 0 & 0 \\ 0 & f_{y} & 0 \\ 0 & 0 & 1\end{array}\right)$ and $\boldsymbol{\Pi}=\left(\begin{array}{cccc}1 & 0 & 0 & 0 \\ 0 & 1 & 0 & 0 \\ 0 & 0 & 0 & 1\end{array}\right)$ where $f_{x}$ and $f_{y}$ define the focal distances in $x$ and $y$ directions of image frame. ${ }^{c} \mathbf{T}_{w}$ denotes a $4 \times 4$ homogeneous matrix that describes the transformation between world and camera frames. It can be further decomposed in a rotation and translation components,

$$
{ }^{c} \mathbf{T}_{w}=\left(\begin{array}{cc}
{ }^{c} \mathbf{R}_{w} & { }^{c} \mathbf{t}_{w} \\
\mathbf{0}_{1 \times 3} & 1
\end{array}\right)
$$

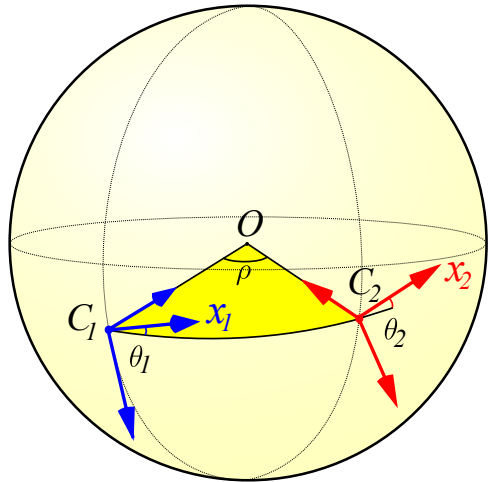

Fig. 2. Geometry of motion between two images taken with SEM (left and right). Transformation between $\mathcal{R}_{1}$ and $\mathcal{R}_{2}$ is a composition of three rotations with angles $\theta_{1}, \rho$, and $\theta_{2}$. Object motion is represented as a motion of camera (SEM).

The fact of parallel projection imposes the following properties on the process of image formation. First, image is invariant to object displacement along the optical axis of the camera. In other words, image is invariant to the distance between camera and object, which is the case in SEM. For instance, moving the sample closer to the electron beam or moving it away will not change the resulting 2D projection.Mathematically, it can be proved as follows. Assume the object has performed a displacement $\Delta Z$ along the optical axis, thus the value of $Q_{z}$ has changed. As it can be seen from the Eq. 1 this value will always be multiplied by zero because of the parallel projection matrix $\Pi$ which has three zeros in the third column. As a result, the depth coordinate is lost in the process of image formation in case of parallel projection.

Secondly, the projection of the object is independent of translations in $x$ and $y$ directions of image frame if the relative coordinates are used both in $3 \mathrm{D}$ object frame and in camera frame [14]. If such translation is performed, only the position of the object in the image changes, but not the disposition of its feature points. For any given reference point $\left(q_{x}^{r}, q_{y}^{r}\right)^{\top}$ in image frame and $\left(Q_{x}^{r}, Q_{y}^{r}, Q_{z}^{r}\right)^{\top}$ in world frame, the expressions for relative coordinates ( $\check{q}$ in image frame and $\check{Q}$ in a world frame) can be written as follows:

$$
\begin{aligned}
&\left(\begin{array}{c}
\check{q}_{x} \\
\check{q}_{y}
\end{array}\right)=\left(\begin{array}{c}
q_{x}-q_{x}^{r} \\
q_{y}-q_{y}^{r}
\end{array}\right) \\
&\left(\begin{array}{c}
\check{Q}_{x} \\
\check{Q}_{y} \\
\check{Q}_{z} \\
1
\end{array}\right)=\left(\begin{array}{c}
Q_{x}-Q_{x}^{r} \\
Q_{y}-Q_{y}^{r} \\
Q_{z}-Q_{z}^{r} \\
1
\end{array}\right)
\end{aligned}
$$

Thus, for every camera, we can choose a reference point defined as the centroid of the set of points detected in this image and furthermore work with relative coordinates. It results in a fact that the centroid of the $3 \mathrm{D}$ points in world frame is projected into the centroid of the points in the image plane. The centroid of $3 \mathrm{D}$ points is considered having coordinates $(0,0,0,1)^{\top}$. 
These two properties allow to draw the following conclusions:

- Parallel projection camera is invariant to translations in image frame, relative coordinates can be used: all detected interest points are translated into $(0,0,1)^{\top}$. It means that the centroid of $3 \mathrm{D}$ points is in $(0,0,0,1)^{\top}$. Therefore, in the relative coordinates, for all taken images $z$-axis of camera is pointing towards the same point, the world origin;

- From the second property, as the object-camera distance is chosen to be one for all views, all camera centers are at the same distance from the world origin.

Thus, when using relative coordinates, at every iteration of movement, the camera lies on the surface of the sphere (Fig. 2). It results in the fact that the following decomposition between two views is possible:

$$
{ }^{2} \mathbf{R}_{1}=\mathbf{R}_{z}\left(\theta_{2}\right) \mathbf{R}_{y}(\rho) \mathbf{R}_{z}^{\top}\left(\theta_{1}\right)
$$

This result is crucial for the purpose of rectification because to obtain the rectified image pair one has to apply a rotation to both image by angles $\theta_{1}$ and $\theta_{2}$, respectively. These angles, slope angles, can be directly derived from the special form of the fundamental matrix as it will be demonstrated further.

\section{METHODS}

The presented algorithm of rectification is based on the properties of epipolar geometry under parallel projection. The next two sections are devoted to the robust estimation of the fundamental matrix for SEM images and to image pair rectification. The developed algorithms do not require any calibration of SEM. They are entirely based on exploitation of the set of correspondences obtained using standard techniques such as SIFT or SURF.

\section{A. Robust estimation of affine fundamental matrix}

In the case of parallel projection the fundamental matrix has the following form:

$$
\mathbf{F}=\left(\begin{array}{ccc}
0 & 0 & a \\
0 & 0 & b \\
c & d & e
\end{array}\right)
$$

where $e$ is often taken as one, $a, b, c, d$ are real numbers. The fundamental matrix is usually estimated from a set of point correspondences obtained using SIFT or SURF feature detectors with further matching. One of the methods dedicated for the estimation of this type of fundamental matrix is the Gold Standard method [9]. It should be noted that one needs at least four point correspondences to find F. The Gold Standard algorithm comprises the following steps. Assume one correspondence is represented by the vector $\mathbf{c}_{i}$ :

$$
\mathbf{c}_{i}=\left(q_{x}^{\prime}, q_{y}^{\prime}, q_{x}, q_{y}\right)^{\top}
$$

Then, in order to work with relative coordinates all points are centered in $(0,0)^{\top}$ :

$$
\check{\mathbf{c}}_{i}=\mathbf{c}_{i}-\overline{\mathbf{c}}
$$

where $\overline{\mathbf{c}}$ is the centroid of points computed as:

$$
\overline{\mathbf{c}}=\frac{1}{N} \sum_{i}^{N} \mathbf{c}_{i}
$$

with $N$ the total number of correspondences found. It allows the construction of $N \times 4$ matrix $\mathbf{A}$ with rows $\check{\mathbf{c}}_{i}^{\top}$. Then, if the singular vector corresponding to the smallest singular value of $\mathbf{A}$ is denoted as $\mathbf{N}$, all five elements of $\mathbf{F}$ can be found using:

$$
\begin{gathered}
(a, b, c, d)=\mathbf{N}^{\top} \\
e=-\mathbf{N}^{\top} \overline{\mathbf{c}}
\end{gathered}
$$

The fundamental matrix is then obtained using Equation (6).

The Gold Standard allows to find such fundamental matrix that minimizes the residual error which represents the mean distance from all points to the corresponding epipolar lines:

$$
\varepsilon_{r}=\frac{1}{N} \sum_{i}^{N}\left[d\left(\mathbf{q}_{i}^{\prime}, \mathbf{F} \mathbf{q}_{i}\right)^{2}+d\left(\mathbf{q}_{i}, \mathbf{F}^{\top} \mathbf{q}_{i}^{\prime}\right)^{2}\right]
$$

where $\mathbf{q}_{i}$ is the $i$-th feature extracted from the first image, $\mathbf{q}_{i}^{\prime}$ from the second image, $d(\cdot, \cdot)$ is the geometrical distance. The main drawback of this method is the lack of robustness towards the presence of outliers. Thus, we use this algorithm inside the Least Median of Squared (LMedS) or RANSAC schemes. Both methods are iterative and based on a random selection on a subset for further model estimation. The goal of LMedS is to minimize the median of the squared errors. It is robust for the sets containing at least $50 \%$ of inliers. RANSAC algorithm seeks to maximize the number of features considered as inliers. In both cases, first, four correspondences are chosen randomly from the whole set as fundamental matrix has four degrees of freedom. Then, the fundamental matrix is estimated using Gold Standard algorithm and the median (LMedS) or the inliers fraction (RANSAC) is compared with the values on previous iterations. These steps are repeated until the lowest median or the maximum number of inliers is found. The final version of the algorithm in case of LMedS scheme is summarized in Fig. 3.

\section{B. Rectification}

The goal of rectification for classical perspective cameras is often stated as follows: apply a perspective transformation to both images in order to make their optical axes parallel and their epipolar lines horizontal. However, in the case of SEM parallel projection with constant magnification, as it will be demonstated further, the condition may be formulated in another way: to obtain a pair of rectified images, the only necessary condition is the coplanarity of $x$ axes of both cameras.

As it can be seen from Fig. 2, there is a circle passing through the centers of both cameras and the world origin. This circle defines uniquely a plane $\pi_{r}$ from three points. As a result, for images to be rectified, it is enough to apply a rotation about optical axis in order to make $x$-axis of both cameras tangent to this circle. As the position of cameras is constrained by the epipolar geometry, the demanded rotation 


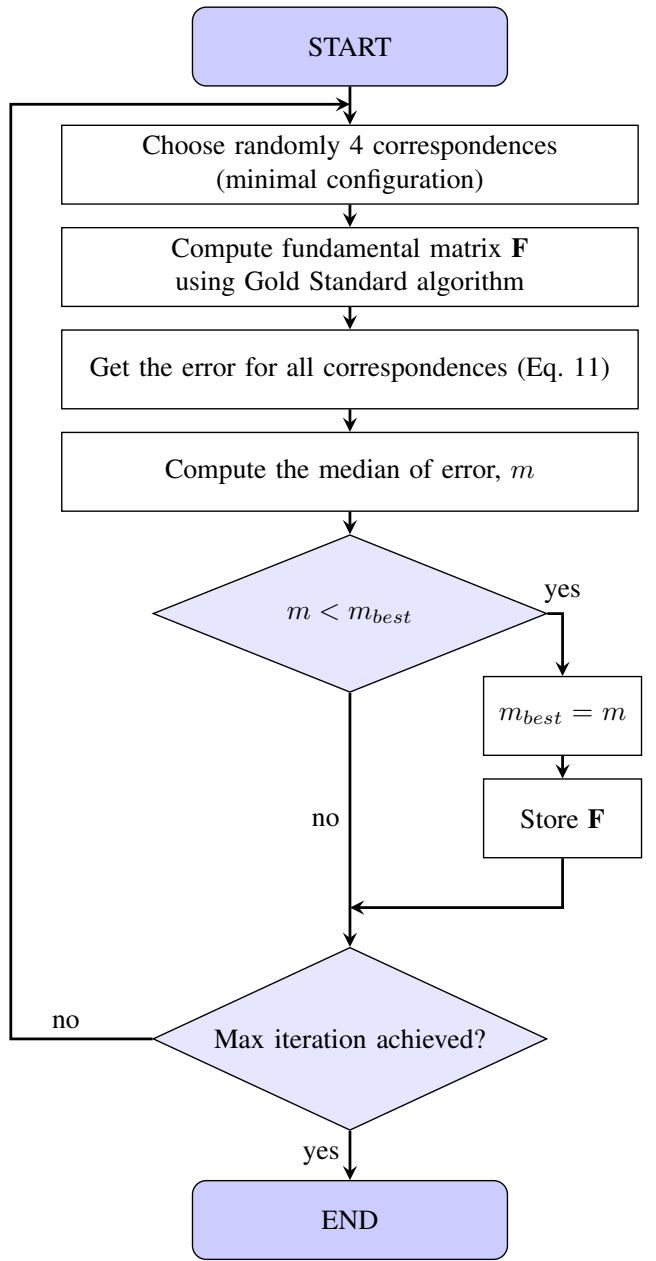

Fig. 3. Algorithm of robust estimation of fundamental matrix for a SEM image pair using Least Median of Squared (LMedS) scheme.

angle can be found using fundamental matrix. In fact, all epipolar lines are parallel between themselves and to the plane $\pi_{r}$, which means that the needed rotation angle can be calculated as a slope of one of epipolar lines for both images:

$$
\theta_{1}=\arctan \left(-\frac{d}{c}\right), \theta_{2}=\arctan \left(-\frac{a}{b}\right)
$$

where $a, b, c, d$ are the elements of the fundamental matrix. Knowing the slope angles, one can rectify the stereo-image using the algorithm presented below.

Next step consists in applying an affine transformation of the same form to both images. The transformation of the first image is:

$$
T_{1}=\left[\begin{array}{lll}
1 & 0 & u \\
0 & 1 & v \\
0 & 0 & 1
\end{array}\right]\left[\begin{array}{ccc}
\cos \left(\theta_{1}\right) & \sin \left(\theta_{1}\right) & 0 \\
-\sin \left(\theta_{1}\right) & \cos \left(\theta_{1}\right) & 0 \\
0 & 0 & 1
\end{array}\right]\left[\begin{array}{ccc}
1 & 0 & -u \\
0 & 1 & -v \\
0 & 0 & 1
\end{array}\right]
$$

which represents the rotation by an angle $\theta$ about the image center $(u, v)^{\top}$. The second transformation $T_{2}$ is obtained by replacing $\theta_{1}$ with $\theta_{2}$. After this transformation all epipolar lines are horizontal, however, they still need to be aligned
TABLE I

\begin{tabular}{|c|c|c|}
\hline & Brassica & $\begin{array}{l}\text { Cutting } \\
\text { tool }\end{array}$ \\
\hline \multicolumn{3}{|l|}{ Images: } \\
\hline size & $854 \times 680$ & $2048 \times 1536$ \\
\hline \multicolumn{3}{|l|}{ Matching: } \\
\hline $\begin{array}{l}\text { total number of features } \\
\text { (with outliers) }\end{array}$ & 138 & 320 \\
\hline \multicolumn{3}{|l|}{ Fundamental matrix: } \\
\hline Residual error (Gold Standard) & 9.159 & 0.555 \\
\hline Residual error (with RANSAC) & 6.168 & 0.105 \\
\hline Residual error (with LMedS) & 0.203 & 0.094 \\
\hline \multicolumn{3}{|l|}{ Rectification } \\
\hline Slope angle $\theta_{1}$, degree & -1.92 & -73.61 \\
\hline Slope angle $\theta_{2}$, degree & -1.83 & -63.69 \\
\hline \multicolumn{3}{|l|}{ Reconstruction } \\
\hline number of points & 406747 & 712588 \\
\hline
\end{tabular}

ALGORITHM PERFORMANCE FOR TWO IMAGE PAIRS

vertically. In order to do that, a vertical shift $\Delta s$ should be applied to one of the image, e.g., if its sign is negative, $\Delta s$ lines should be added to the beginning of the second (right) image.

$$
\Delta s=\frac{1}{N}\left[\begin{array}{lll}
0 & 1 & 0
\end{array}\right] \sum_{i}^{N}\left[T_{1} q_{i}-T_{2} q_{i}{ }^{\prime}\right]
$$

At this step, the images are rectified: all epipolar lines are horizontal after image rotation and then the vertical shift was compensated.

\section{RESULTS}

In the present work, two image pairs are choosen to present the results:

- Brassica [15], [16], [17], [18], [19]: Images of a pollen grain of white turnip plant from a Hitachi S-4800 field emission SEM. Authors present their results of sparse reconstruction (up to 1000 points). $\rho=3$ degrees.

- Cutting tool: Two images of the edge of micro cutting tool. Images were taken using a SEM Zeiss AURIGA 60. $\rho=5$ degrees.

The preliminary step for the techniques presented in this paper consists in extraction of the features with further matching. For the feature detection and extraction the AKAZE algorithm was used [20]: the authors of this algorithm declare that it outperforms BRISK, ORB, SURF and SIFT in most of the scenarios. When the features are extracted, a matching strategy should be adopted: in the present work a matching was realized using BruteForce algorithm implemented in OpenCV library [21]. The analysis of the results showed that the set of correspondences contains outliers, that were than partially filtered using the method presented by Lowe in [1]: it rejects poor matches by computing the ratio between the best and second-best match. If the ratio is below a defined threshold, the match is discarded as being of low-quality. The value of matching threshold was kept the same for all image pairs. This step allowed to guarantee that the set contains at least $50 \%$ of inliers. The number of matches obtained is 138 and 
Original image pairs:

Magnification: 10K

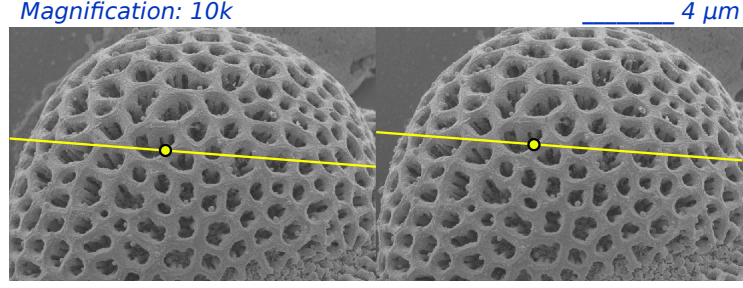

Rectified image pairs:

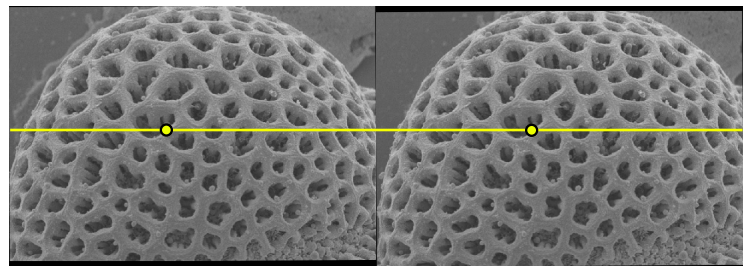

Disparity maps:

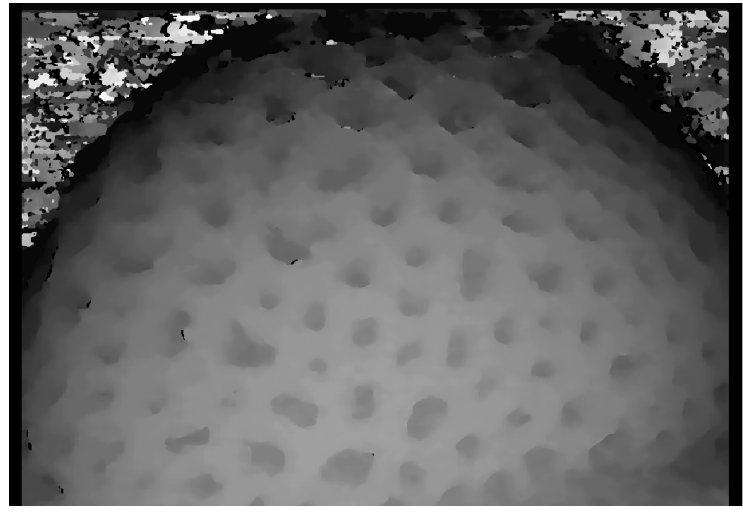

Dense point clouds:

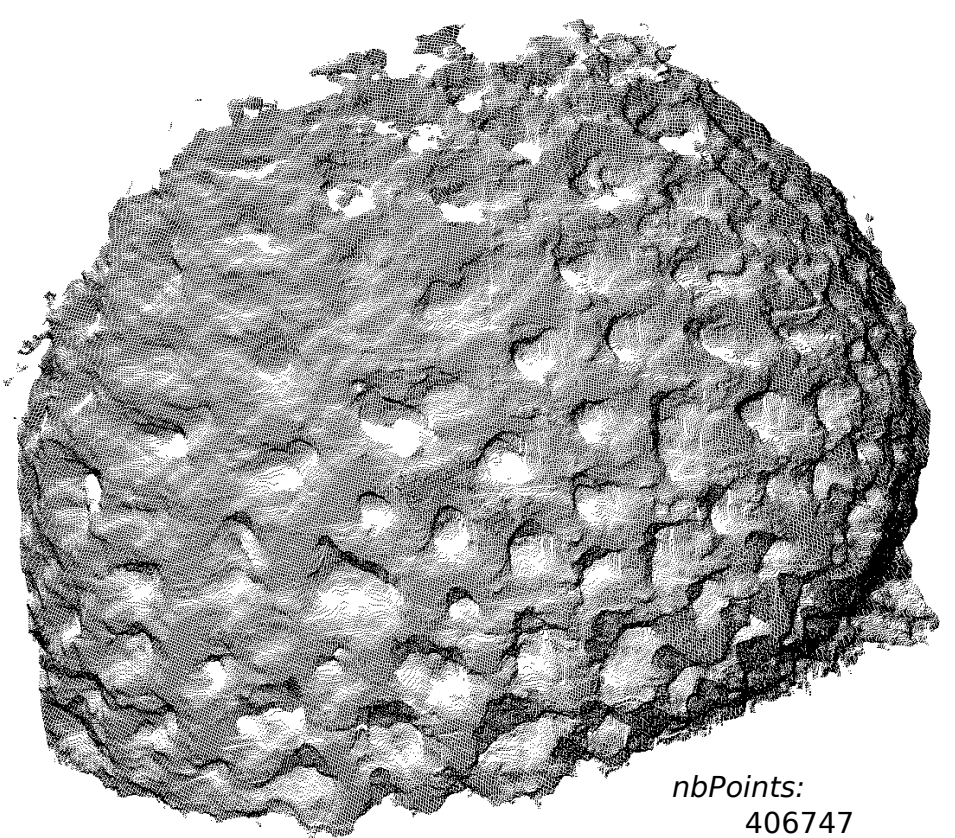

Magnification: $2 k$

$10 \mu \mathrm{m}$
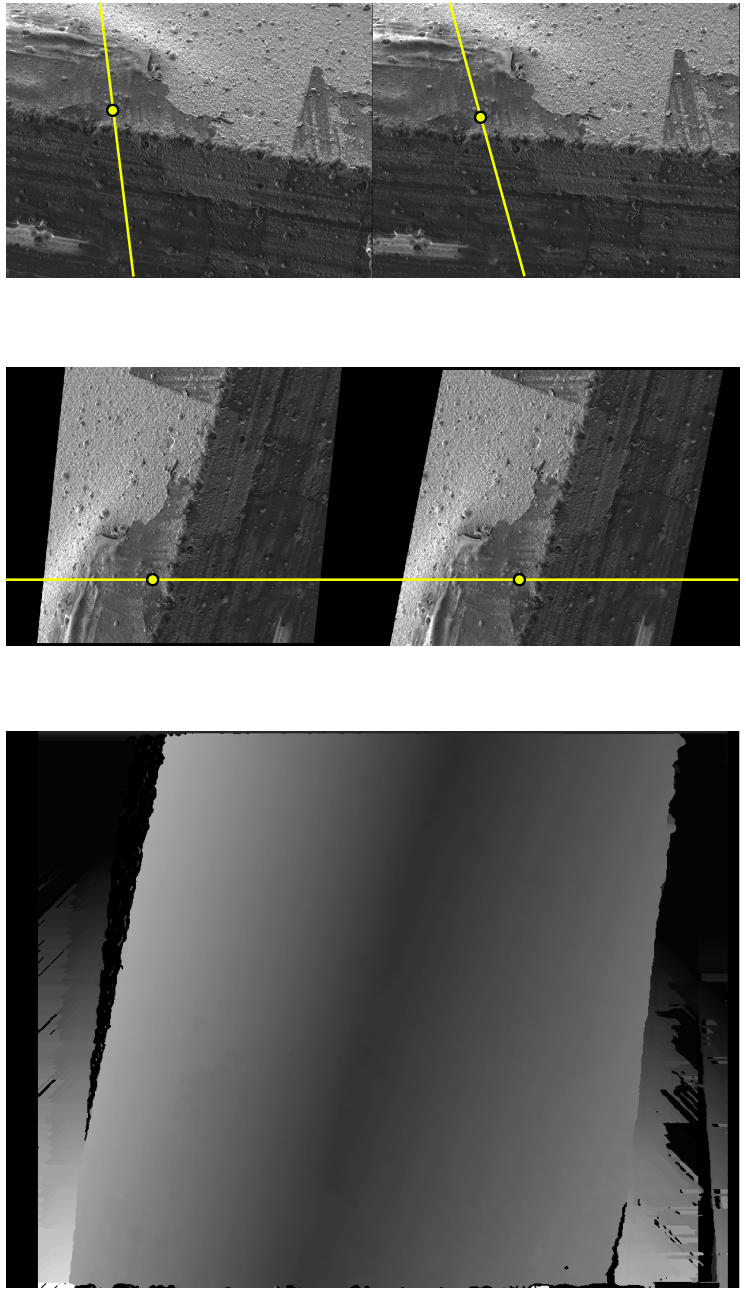

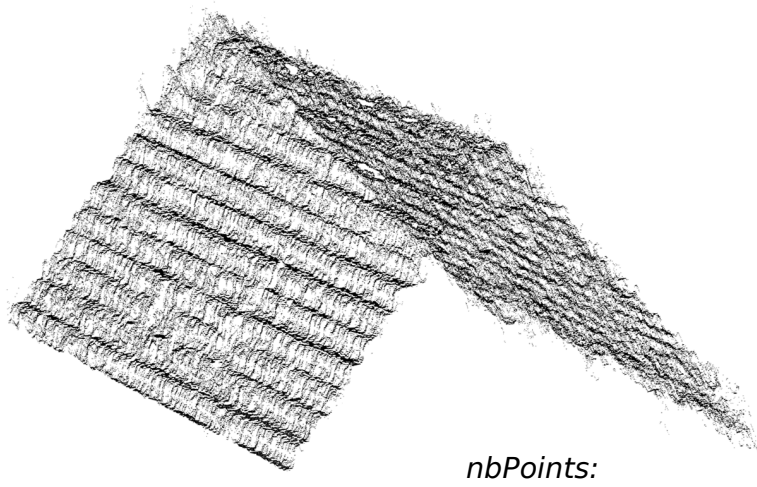

712588

Fig. 4. Results of dense 3D reconstruction in SEM. Left column: Brassica pollen grain. Right column: edge of cutting tool. For each pair, only one of epipolar lines is shown for clearness. 
320 for Brassica and Cutting tool, respectively. All results are summarized in Table I.

The comparison of fundamental matrix estimation proved the viability of the introduction of robust estimators such as RANSAC and LMedS. The number of iteration was kept the same and equal to 1000. In the case of RANSAC, a threshold was defined with the value of 0.1 pixels squared. The residual error is almost ten times smaller in the case of LMedS scheme. As it was already mentioned before, the LMedS results were chosen for rectification as there is no need in threshold definition.

Regarding the rectification, the slope angles computed from the fundamental matrices are: -1.92 and -1.83 degrees for Brassica dataset and -73.61 and -63.69 degrees for Cutting tool. The original as well as the rectified image pairs are shown in Fig. 4. Disparity maps were obtained using the technique of Semi-Global matching implemented in OpenCV software [22]. Their quality validates the rectification approach presented in this paper. Finally, the reconstructed point clouds were obtained from disparity maps and known camera matrices using the triangulation techniques [23]. The number of points has increased dramatically in comparison with sparse reconstruction, it was multiplied by a factor of 1000: 406747 and 712588 for Brassica and Cutting tool, respectively.

\section{CONCLUSion}

Dense 3D reconstruction is one of the few tools allowing to measure the 3D properties of microscopic objects. In order to achieve it, the images need to be rectified first to proceed to dense matching algorithms. In this paper, we address the problem of rectification for an uncalibrated SEM for which the image formation is described with parallel projection model. First, we propose an algorithm allowing accurate and robust measurement of fundamental matrix describing the geometry of camera/object movement. The estimated matrix has smaller error $(\times 10)$ comparing to literature solution that was possible due the robustness towards the presence of outliers in correspondence set. Secondly, a direct linear method of rectification, based on the camera properties and fundamental matrix, is proposed. Both algorithms were then validated on two image pairs coming from two different SEMs: disparity maps as well as the results of dense 3D reconstruction were obtained.

\section{ACKNOLEDGMENT}

This work has been supported by the Labex ACTION project (contract ANR-11-LABX-0001-01), Equipex ROBOTEX project (contract ANR-10-EQPX-44-01), the cross border project CITHADEL of Interreg France-Switzerland program through the European regional development fund. Authors would like to thank ChronoEnvironment Lab (Besançon, France) for providing samples of pollen grains, Department of Applied Mechanics (FEMTO-ST Institute) for providing the cutting tool specimen, Patrick Rougeot ${ }^{1}$ and Olivier Lehmann ${ }^{1}$ for their help with sample preparation and image acquisition.

\section{REFERENCES}

[1] D. G. Lowe, "Distinctive image features from scale-invariant keypoints," International journal of computer vision, vol. 60, no. 2, pp. 91-110, 2004.

[2] H. Bay, A. Ess, T. Tuytelaars, and L. Van Gool, "Speeded-up robust features (surf)," Computer vision and image understanding, vol. 110, no. 3, pp. 346-359, 2008

[3] L. Zhang, D. Zhu, R. Wu, E. Liu, and D. Zhang, "Tool wear investigation using sem 3d reconstruction technique," in Int. Conf. on Digital Manufacturing and Automation (ICDMA). IEEE, 2011, pp. 1364-1367.

[4] C. Loop and Z. Zhang, "Computing rectifying homographies for stereo vision," IEEE Conference on Computer Vision and Pattern Recognition, vol. 1, 1999.

[5] M. Pollefeys, R. Koch, and L. Van Gool, "A simple and efficient rectification method for general motion," in Computer Vision, 1999. The Proceedings of the Seventh IEEE International Conference on, vol. 1. IEEE, 1999, pp. 496-501.

[6] N. Cornille, D. Garcia, M. A. Sutton, S. McNeill, and J.-J. Orteu, "Automated 3-d reconstruction using a scanning electron microscope," in SEM conference on experimental and applied mechanics, 2003.

[7] O. Sinram, M. Ritter, S. Kleindick, A. Schertel, H. Hohenberg, and J. Albertz, "Calibration of a sem, using a nanopositioning tilting table and a microscopic calibration pyramid," International Archives of Photogrammetry Remote Sensing and Spatial Information Sciences, vol. 34, no. 5, pp. 210-215, 2002.

[8] L. Cui and E. Marchand, "Calibration of scanning electron microscope using a multi-image non-linear minimization process," in Robotics and Automation (ICRA), 2014 IEEE International Conference on. IEEE, 2014, pp. 5191-5196.

[9] R. Hartley and A. Zisserman, Multiple view geometry in computer vision. Cambridge university press, 2003.

[10] A. Fusiello, E. Trucco, and A. Verri, "A compact algorithm for rectification of stereo pairs," Machine Vision and Applications, vol. 12, no. 1, pp. 16-22, 2000.

[11] S. Kumar, C. Micheloni, C. Piciarelli, and G. L. Foresti, "Stereo rectification of uncalibrated and heterogeneous images," Pattern Recognition Letters, vol. 31, no. 11, pp. 1445-1452, 2010.

[12] Z. Zhang, "Flexible camera calibration by viewing a plane from unknown orientations," in Computer Vision, 1999. The Proceedings of the Seventh IEEE International Conference on, vol. 1. IEEE, 1999, pp. 666-673.

[13] S. Liansheng, Z. Jiulong, and C. Duwu, "Image rectification using affine epipolar geometric constraint," Journal of Software, vol. 4, no. 1, p. 27, 2009.

[14] L. Quan, "Self-calibration of an affine camera from multiple views," International Journal of Computer Vision, vol. 19, no. 1, pp. 93-105, 1996.

[15] A. P. Tafti, "3D SEM surface reconstruction: An optimized, adaptive and intelligent approach," PhD thesis, 2016.

[16] A. P. Tafti, J. Holz, A. Baghaie, H. Owen, M. M. He, and Z. Yu, "3DSEM++: Adaptive and intelligent 3D SEM surface reconstruction," Micron, vol. 87, pp. 33-45, 2016.

[17] A. P. Tafti, A. B. Kirkpatrick, J. D. Holz, H. A. Owen, and Z. Yu, "3DSEM: A 3D microscopy dataset," Data in Brief, 2015.

[18] A. P. Tafti, A. B. Kirkpatrick, Z. Alavi, H. A. Owen, and Z. Yu, "Recent advances in 3d sem surface reconstruction," Micron, vol. 78, pp. 54-66, 2015.

[19] A. P. Tafti, A. Kirkpatrick, H. Owen, and Z. Yu, "3d microscopy vision using multiple view geometry and differential evolutionary approaches," in Advances in Visual Computing. Springer, 2014, pp. 141-152.

[20] P. F. Alcantarilla, J. Nuevo, and A. Bartoli, "Fast explicit diffusion for accelerated features in nonlinear scale spaces," IEEE Trans. Patt. Anal. Mach. Intell, vol. 34, no. 7, pp. 1281-1298, 2011.

[21] G. Bradski, "OpenCV library," Dr. Dobb's Journal of Software Tools, 2000.

[22] S. Dröppelmann, M. Hueting, S. Latour, and M. van der Veen, "Stereo vision using the opencv library," 2010.

[23] R. I. Hartley and P. Sturm, "Triangulation," Computer vision and image understanding, vol. 68, no. 2, pp. 146-157, 1997. 